\title{
Impact of molecular structure on the lubricant squeeze-out between curved surfaces with long range elasticity
}

Tartaglino, Ugo; Sivebæk, Ion Marius; Persson, B N J; Tosatti, E

Published in:

Journal of Chemical Physics

Link to article, DOI:

$10.1063 / 1.2210008$

Publication date:

2006

Document Version

Publisher's PDF, also known as Version of record

Link back to DTU Orbit

Citation $(A P A)$ :

Tartaglino, U., Sivebæk, I. M., Persson, B. N. J., \& Tosatti, E. (2006). Impact of molecular structure on the lubricant squeeze-out between curved surfaces with long range elasticity. Journal of Chemical Physics, 125(1), 014704. https://doi.org/10.1063/1.2210008

\section{General rights}

Copyright and moral rights for the publications made accessible in the public portal are retained by the authors and/or other copyright owners and it is a condition of accessing publications that users recognise and abide by the legal requirements associated with these rights.

- Users may download and print one copy of any publication from the public portal for the purpose of private study or research.

- You may not further distribute the material or use it for any profit-making activity or commercial gain

- You may freely distribute the URL identifying the publication in the public portal 


\title{
Impact of molecular structure on the lubricant squeeze-out between curved surfaces with long range elasticity
}

\author{
U. Tartaglino ${ }^{a)}$ \\ IFF, FZ-Jülich, D-52425 Jülich, Germany and International School for Advanced Studies (SISSA) \\ and INFM Democritos National Simulation Center, Via Beirut 2-4, I-34014 Trieste, Italy
}

I. M. Sivebaek

IFF, FZ-Jülich, D-52425 Jülich, Germany; Novo Nordisk A/S, Research and Development, DK-3400 Hillerød, Denmark; and MEK-Energy, Technical University of Denmark, DK-2800 Lyngby, Denmark

B. N. J. Persson

IFF, FZ-Jülich, D-52425 Jülich, Germany

\section{E. Tosatti}

International School for Advanced Studies (SISSA) and INFM Democritos National Simulation Center, Via Beirut 2-4, I-34014 Trieste, Italy and The Abdus Salam International Centre for Theoretical Physics (ICTP), P.O. Box 586, I-34014 Trieste, Italy

(Received 27 March 2006; accepted 9 May 2006; published online 5 July 2006)

\begin{abstract}
The properties of butane $\left(\mathrm{C}_{4} \mathrm{H}_{10}\right)$ lubricants confined between two approaching solids are investigated by a model that accounts for the curvature and elastic properties of the solid surfaces. We consider the linear $n$-butane and the branched isobutane. For the linear molecule, well defined molecular layers develop in the lubricant film when the width is of the order of a few atomic diameters. The branched isobutane forms more disordered structures which permit it to stay liquidlike at smaller surface separations. During squeezing the solvation forces show oscillations corresponding to the width of a molecule. At low speeds $(<0.1 \mathrm{~m} / \mathrm{s})$ the last layers of isobutane are squeezed out before those of $n$-butane. Since the (interfacial) squeezing velocity in most practical applications is very low when the lubricant layer has molecular thickness, one expects $n$-butane to be a better boundary lubricant than isobutane. With $n$-butane possessing a slightly lower viscosity at high pressures, our result refutes the view that squeeze-out should be harder for higher viscosities; on the other hand our results are consistent with wear experiments in which $n$-butane were shown to protect steel surfaces better than isobutane. (C) 2006 American Institute of Physics.
\end{abstract}

[DOI: $10.1063 / 1.2210008]$

\section{INTRODUCTION}

Modern materials are subject to increasing loads and are used under still more demanding conditions. This emphasizes the need for a better understanding of friction, lubrication, and wear phenomena. ${ }^{1,2}$

An example of the above is the fuel lubricated diesel engine injection pump, capable of delivering in excess of 2000 bars today compared to 500 bars 15 years ago. This increase in injection pressure has ensured higher engine efficiencies and lower pollution levels.

Historically the diesel oil sulfur reduction both in the 1960s and 1990s combined with the high pressures has played a significant role in injection pump durability. Sulfur, along with other polarity inducing atoms, is part of polar species that ensure low wear in boundary lubrication, a predominant regime in pumps lubricated by diesel oil. Today there exist several accelerated laboratory tests capable of predicting the lubricating abilities of a diesel oil. If a fuel fails such a test, lubricity additives in small proportions are added to ensure proper wear resistance.

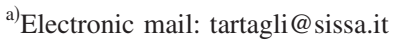

The currently used diesel oil wear test in Europe is the high frequency reciprocating rig (HFRR) which is covered by several standards. ${ }^{3,4}$ The principle in the HFRR is the sliding of a fixed steel ball, loaded with $2 \mathrm{~N}$ on a steel disk for $75 \mathrm{~min}$. The motion is reciprocating with a stroke of $1 \mathrm{~mm}$ and a frequency of $50 \mathrm{~Hz}$. As the specimen contact is fuel lubricated the resulting wear scar diameter on the ball expresses the lubricity of the tested fuel. The configuration of the HFRR is shown in Fig. 1.

The HFRR ability of predicting the lifetime of injection pumps has been questioned when low-viscosity fuels are tested. It appears that a decrease in fuel viscosity requires an increase in lubricity (smaller wear scar) to ensure full lifetime of the pumps. This relation between viscosity and lubricity was confirmed as a new fuel appeared in the 1990s: dimethyl ether (DME). This fuel has a viscosity 20 times lower than that of diesel oil and it was established that an adequate lubricity level according to the HFRR standards was not sufficient to protect the pump surfaces. ${ }^{5-7}$

A study ${ }^{8}$ has argued that fluid viscosity is only a secondary property in the HFRR. Based on molecular dynamics calculations it was discovered that the squeeze-out of linear alkanes from surface contacts is primarily a function of the 


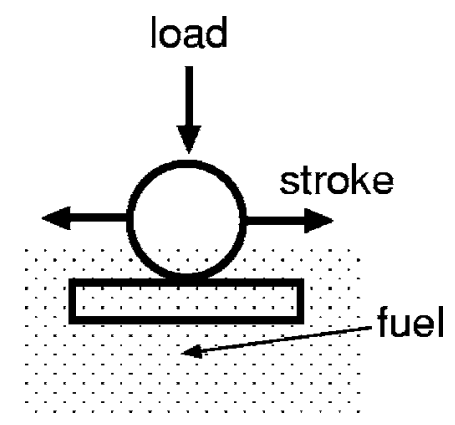

FIG. 1. The ball on disk configuration in a HFRR wear test.

length of the molecule. Experimental data shown in Fig. 2 demonstrate that branched alkanes perform worse in the HFRR than their linear isomers although their viscosities are almost the same. ${ }^{6}$ This observation confirms that the bulk viscosity may be a secondary property in boundary lubrication.

The friction and wear properties in boundary lubrication of lubricants with branched and linear molecules have been investigated both experimentally ${ }^{9-12}$ and using theoretical simulation methods. ${ }^{13-17}$

Whether linear alkanes or their branched isomers are the best lubricants according to the cited literature remains uncertain. A number of studies indicate that $n$-alkanes are the best lubricants, ${ }^{13,14,16,17}$ whereas others claim that branched alkanes perform better. ${ }^{9,11,12,15}$ One major weakness of the published studies is that the lubricant squeezing is stopped at a separation of about $8 \AA$ between the surfaces. At this point there are still a few monolayers left protecting the surfaces, and significant wear originating from cold welding should only appear when the last lubricant layer is squeezed out. ${ }^{18}$

In the present computer simulation study we investigate theoretically the lubrication abilities of $n$ - and isobutane extending the squeezing down to a surface separation of $0 \AA$ so that the important expulsion of the last monolayer is addressed.

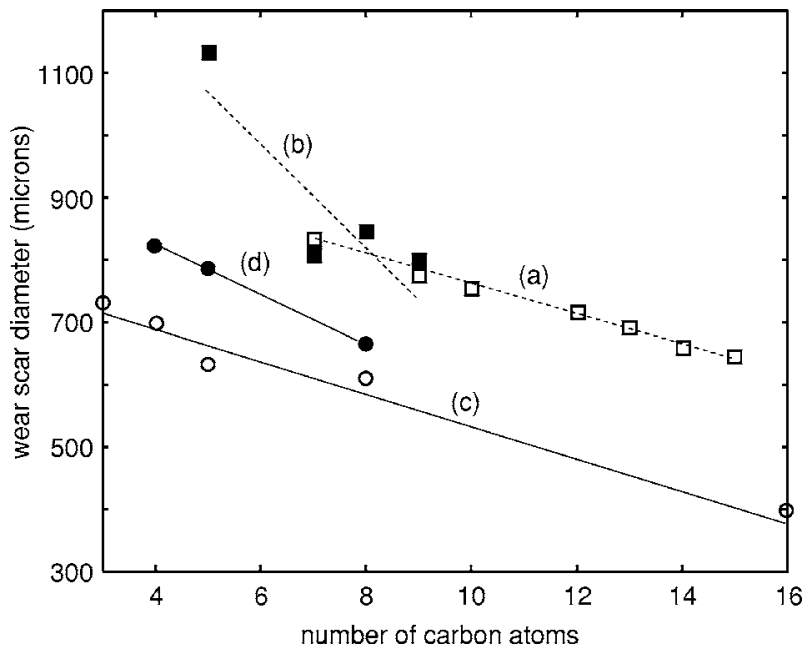

FIG. 2. The wear scar diameter for various alkanes as a function of the molecular length. The squares are from Ref. 19 and the circles from Ref. 8. (a) and (c) are for linear alkanes and (b) and (d) for branched isomers.

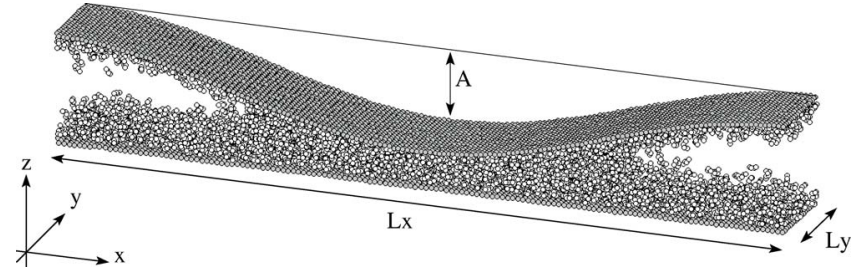

FIG. 3. The simulated sample: two elastic walls with lubricant in between are pushed together. The sinusoidal profile of the upper surface generates a line contact in the middle, where lubricant forms a neck and eventually it is squeezed out by the load. Periodic boundary conditions are applied along $x$ and $y$.

\section{THE MODEL}

Figure 3 shows the geometry for the simulation of the squeeze-out process. Lubricant molecules are confined between two elastic walls. The lower wall, or substrate, is flat, while the upper one, called block, has a sinusoidal profile, so that a contact region appears in the center of the sample. The liquid lubricant adheres to the walls, forming a neck in the central region. When the block is pushed towards the substrate, the lubricant has space to expand laterally into the vapor. Periodic boundary conditions are applied along the $x$ and $y$ directions. The periodically repeated cell forms a rectangle $L_{x} \times L_{y}$, with $L_{x}=416 \AA$ and $L_{y}=52 \AA$. The contact region is not circular, but forms an infinite long strip parallel to the $y$ axis.

The lubricant molecules are described through the optimized potential for liquid simulation (OPLS) ${ }^{20,21}$ this potential is known to provide density and viscosity of hydrocarbons close to the experimental one.

Each butane molecule comprises four units (particles), each particle corresponding to one chemical group $\mathrm{CH}_{3}$, $\mathrm{CH}_{2}$, or $\mathrm{CH}$ (united atom model). The interaction between particles of different molecules is described by LennardJones potentials. The intramolecular interactions include two body forces that keep the bond length $\mathrm{C}-\mathrm{C}$ close to $1.53 \AA$, three body forces imposing a preferred angle of $115^{\circ}$ between the carbon atoms, and four body forces favoring a well defined torsion of the molecules. The four body forces apply to the sequence of carbon atoms $\mathrm{C}-\mathrm{C}-\mathrm{C}-\mathrm{C}$; thus they are present only for $n$-butane.

For the isobutane molecules we introduced also an antiumbrella inversion potential, analogous to what has already been done by Mondello and Grest ${ }^{22}$ for branched hydrocarbons; the only difference here being that in the isobutane molecule it is not possible to distinguish which carbon atom constitutes the lateral branch and which constitute the backbone of the molecule. Therefore we symmetrized the antiinversion potential.

The interaction between the beads of the lubricant molecule and the walls' atoms is also given by Lennard-Jones forces, whose energy parameter $\epsilon_{0}=18.6 \mathrm{meV}$, as in Ref. 23, yielding an adsorption energy of about $0.3 \mathrm{eV} /$ molecule, comparable to that of butane on $\mathrm{Au}(111) .^{24}$

The elastic energy due to the walls' deformation is also involved in the nucleation process that triggers the transition from $n$ to $n-1$ monolayers of lubricant in the confined regime. ${ }^{25}$ In principle, we can achieve a realistic description 
of the walls' elastic response by simulating thick walls comprising many layers. Since we necessitate proper elastic behavior up to wavelengths comparable with the size of the contact area the walls' thickness must not be smaller that the lateral size of the box, $L_{y}=52 \AA$. This indeed would imply too many atoms, slowing down the calculations. Instead we adopted the model described in Ref. 26: for each wall only the outermost layer of atoms is considered; these atoms are connected to a rigid surface with springs that take into account both the compressibility and the shear rigidity of the wall. Similar springs connect together the square grid of atoms of the wall. The advantage of this approach is double: with a relatively small number of atoms it is possible to describe the long range elasticity of the walls and to impose a curved profile simply by using a curved rigid surface.

The rigid surface connected to the substrate's atoms is flat and its position is fixed. The upper rigid surface has the profile

$$
z(x, y)=z_{0}+\frac{A}{2}\left(1-\cos \left(\frac{2 \pi x}{L_{x}}\right)\right),
$$

where the amplitude of corrugation $A$ is 20,40 , or $60 \AA$. The case $A=20 \AA$ guarantees a larger contact region, but it does not allow enough empty space (or rather vapor space region) for the squeeze-out of the last two layers; it was used only for comparison with the other cases to ensure that the size of the contact region is large enough for the nucleation of the squeeze-out process.

The springs connecting the walls' atoms to the rigid surfaces simulate the elastic response of a gold film of thickness $50 \AA$, comparable with the lateral size $L_{y}$ of the sample.

The substrate consists of $144 \times 18$ atoms in a square lattice with lattice spacing of $2.889 \AA$; the block layer is made of $160 \times 20$, atoms with lattice spacing of $2.6 \AA$. The different lattice spacing is to reduce the commensurability between the walls. Both substrate's and block's atoms have the same mass of gold: $197 \mathrm{amu}$. In most of the simulations we employed 2800 lubricant molecules, which deposit on the two walls forming about two monolayers of adsorbate on each surface.

\section{SIMULATION RESULTS}

Two simulations are prepared in parallel for iso- and $n$-butane with identical conditions. Initially the whole system is thermalized and the lubricant adheres to the two walls. Then the rigid upper surface is moved down towards the substrate with constant speed, i.e., $z_{0}$ in Eq. (1) is constrained to decrease linearly with time. As the lubricant layers on the two walls get in contact, a neck is formed in the contact area. The lubricant clearly shows layering both for the $n$-butane and for the more disordered isobutane (Fig. 4). The change in the number of monolayers takes place abruptly, with a sudden decrease of pressure due to the relaxation of the elastic energy stored in the walls.

Figure 5 shows the behavior of pressure versus time while squeezing at a speed of $1 \mathrm{~m} / \mathrm{s}$ and at a temperature of $300 \mathrm{~K}$. The $y$ axis contains a spatial average of the pressure, that is the vertical force divided by the area $L_{x} L_{y}$ of the

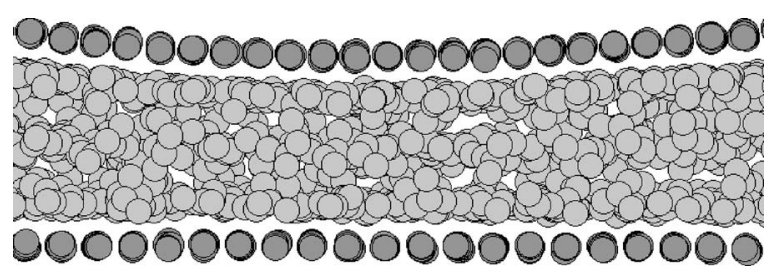

FIG. 4. The confined regime: the liquid lubricant gets ordered into 2D layers when it is confined between atomically flat surfaces. The picture shows the side view of isobutane in the contact region between the two walls. Light balls represent $\mathrm{CH}$ and $\mathrm{CH}_{3}$ groups.

simulated cell. The contact region is about $15 \%$ of the whole cell size, and the real pressure in the center of the contact area is much larger than the average pressure. Negative pressures observed in some time intervals are due to the attractive capillarity forces of the neck of lubricant. The plotted curves show that isobutane is squeezed out earlier and at a lower pressure. This is the typical behavior we observed when there is more than one monolayer. Conversely the removal of the very last monolayer appears to be easier for $n$-butane. As can be observed in Fig. 6, the ejection of the isobutane not only happens later, but it is slower and a small group of seven molecules remains trapped at the end. This suggests that the simulated squeeze-out of the last layer is strongly affected by dynamical effects, such as the drag friction when the lubricant slides on the solid walls. Thus, even though the nucleation barrier of the hole may be smaller for isobutane, the simulated system might not have enough time to exit its metastable state before the pressure is further increased by the vertical (squeezing) motion of the block.

The behavior at higher temperatures is qualitatively the same. At $T=350 \mathrm{~K}$ the switch from three to two monolayers and from two to one monolayer happens first for isobutane, but the very last layer of isobutane stays longer between the walls, and five isobutane molecules remain trapped there (see Fig. 7). Another independent simulation at $T=330 \mathrm{~K}$ (not shown) confirms this trend.

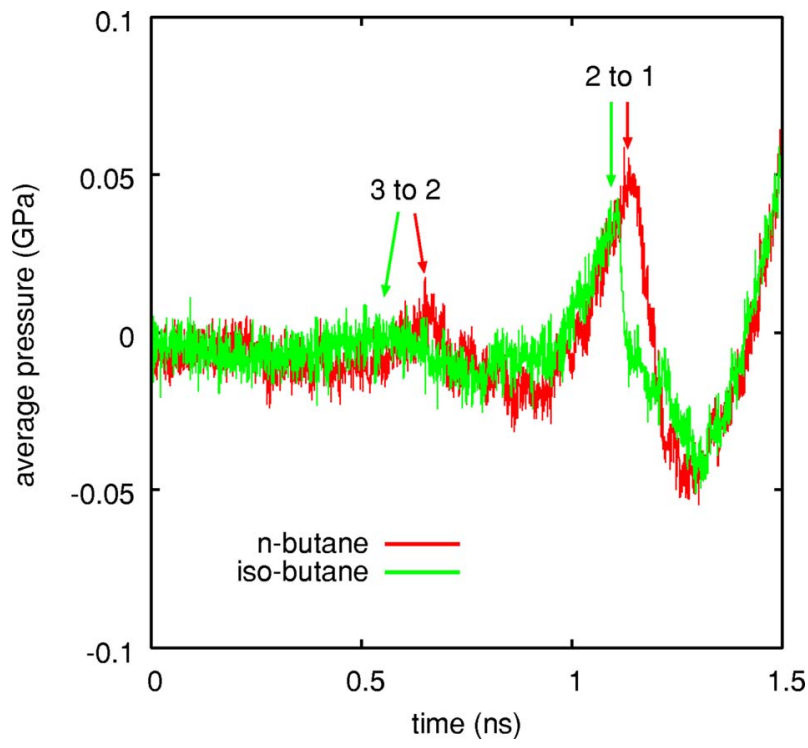

FIG. 5. Squeeze-out of butane at $T=300 \mathrm{~K}$. The pressure drops when the number of layers switches from 3 to 2 and from 2 to 1 . Squeezing speed $=1 \mathrm{~m} / \mathrm{s}$. 


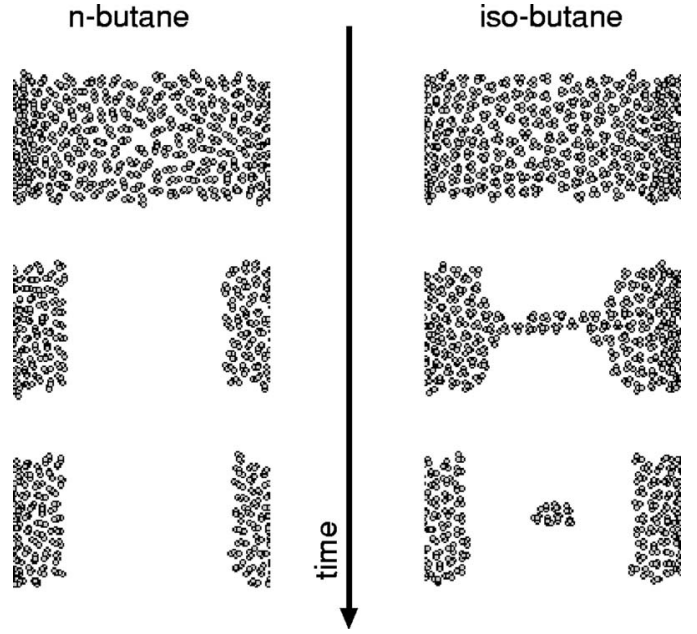

FIG. 6. Top view of the lubricant molecules in the central part of the contact area immediately before the expulsion of the last monolayer (top), after $0.5 \mathrm{~ns}$ (middle), and after $1 \mathrm{~ns} . T=300 \mathrm{~K}$, squeezing speed $=1 \mathrm{~m} / \mathrm{s}$. (Block and substrate are parallel to the plane of the figure.)

The transition from two to one isobutane monolayer reveals another interesting feature: the pressure drops down in two stages, as is shown in the plot of Fig. 7 for time close to $1.2 \mathrm{~ns}$. What happens is clearly illustrated in the snapshots of Fig. 8: initially there is the squeeze-out of one of the two layers. The remaining monolayer does not have a preferential orientation of the molecules parallel to the walls; the plateau in the pressure versus time graph at $t \approx 1.2 \mathrm{~ns}$ is due to this thick monolayer. Finally the molecules change their orientation forming a thinner monolayer, yielding a smaller second pressure drop. This kind of two-stage squeezing of the second layer has been observed in other simulations too, but only for isobutane. It is, in fact, a steric effect due to the shape of the molecule. Similar pressure-induced phase transitions have been observed in other computer simulations of squeeze-out. Thus, in Ref. 27 it was shown that Xe atoms

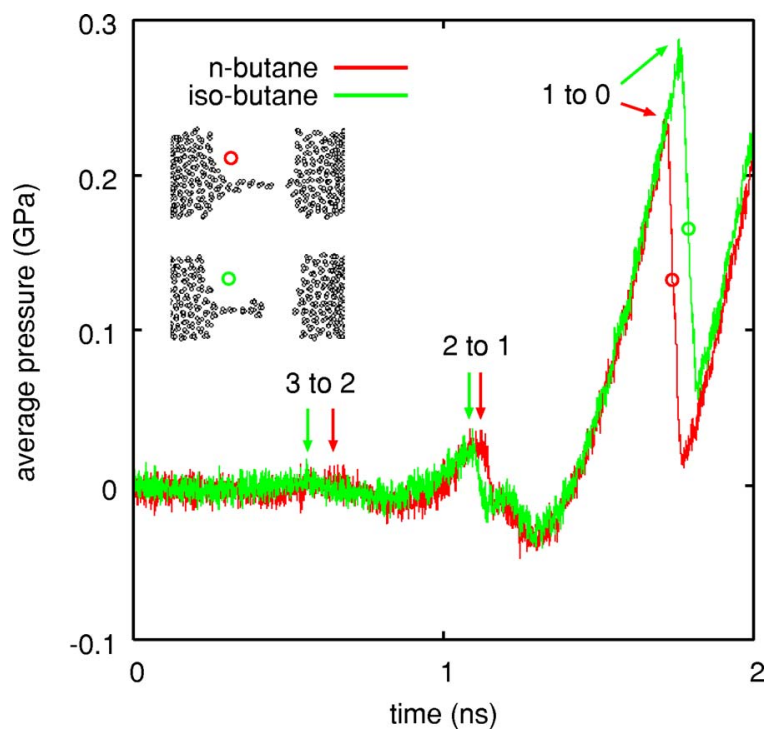

FIG. 7. Pressure vs time during the complete squeeze-out of three monolayers at $T=350 \mathrm{~K}$, squeezing speed $=1 \mathrm{~m} / \mathrm{s}$. The pictures show the lubricant while the last monolayer is being removed. Corrugation of the upper profile: $A=60 \AA$; contact area: $10 \%$ of the cell's area.
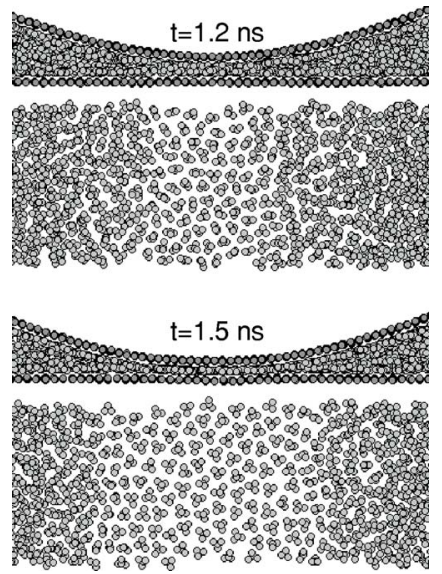

FIG. 8. Side and top views of the isobutane monolayer at different times (and pressures). Images refer to the simulation of Fig. 7.

between two solid surfaces exhibited triangular bilayers at low squeezing pressure, which abruptly transformed to fcc(100) layers parallel to the solid surfaces when the pressure increased. This transition allowed the surfaces to move closer to each other which released elastic energy.

The simulations at lower squeezing speed give clear evidence that the relatively higher lubricity of the last layer of isobutane is a kinetic effect. Thus, at $T=300 \mathrm{~K}$ and for the compression speed of $1 \mathrm{~m} / \mathrm{s}$ (Fig. 6) $n$-butane is removed first, while when the speed is $0.1 \mathrm{~m} / \mathrm{s}$ the removal of the two lubricants happens almost at the same time (not shown). Finally when the squeezing is carried at the speed of $0.03 \mathrm{~m} / \mathrm{s}$ isobutane is removed first and at a lower pressure (Fig. 9). In this case there is still some small island of molecules trapped, shown in the picture, confirming once more that the lateral sliding of isobutane is slower.

A further evidence that the removal of the last monolayer is influenced by kinetic effects comes from the squeez-
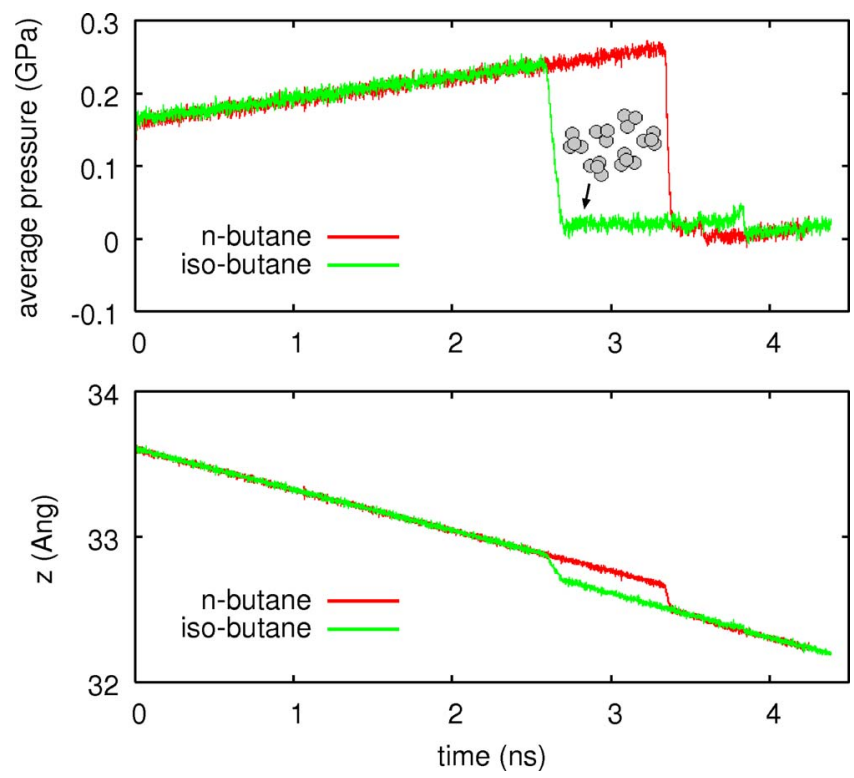

FIG. 9. Last monolayer squeeze-out at low speed. $T=300 \mathrm{~K}$, squeezing speed $=0.03 \mathrm{~m} / \mathrm{s}$. Isobutane is removed earlier at a lower pressure, but the cluster of six molecules shown in the picture remains trapped up to $t$ $=3.6$ ns. Below: the average position of the upper wall's atoms vs time. 


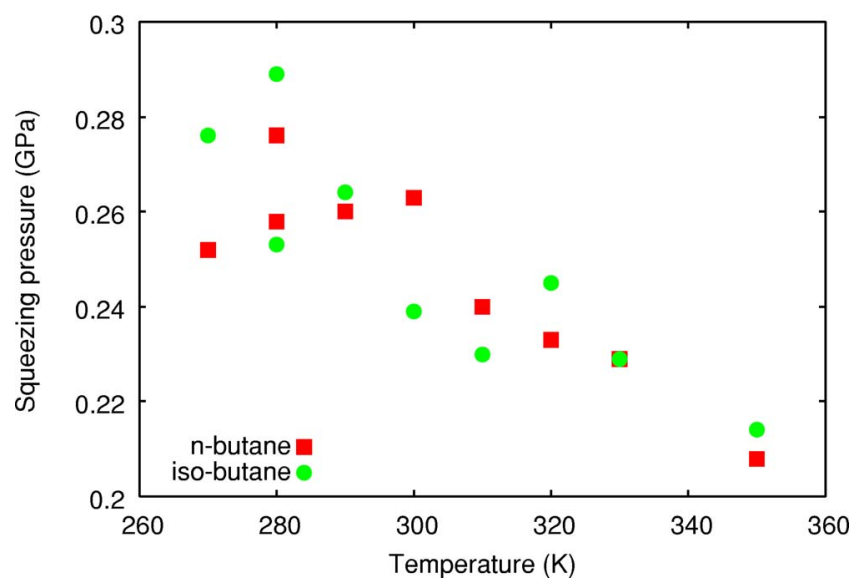

FIG. 10. Pressure required to squeeze-out the last lubricant layer while pushing at the speed of $0.03 \mathrm{~m} / \mathrm{s}$. Data refer to the spatial average over the whole simulation cell. The pressure in the center of the contact area is much larger. Each point is the result of a single independent simulation, requiring about two months of CPU time on a standard PC.

ing pressure versus pushing speed $v_{z}$ : keeping all the other conditions identical, we observed that the pressure at which the last layer is removed does decrease when the block is pushed down slowly. For example, for isobutane at $T$ $=300 \mathrm{~K}$ we obtained the following pressures: $0.340 \mathrm{GPa}$ when $v_{z}=1 \mathrm{~m} / \mathrm{s}, \quad 0.257 \mathrm{GPa}$ when $v_{z}=0.1 \mathrm{~m} / \mathrm{s}$, and $0.239 \mathrm{GPa}$ when $v_{z}=0.03 \mathrm{~m} / \mathrm{s}$ (as usual, these are spatial averages, the true pressures in the contact area being much higher). Similarly, the corresponding simulations for $n$-butane gave $0.321,0.252$, and $0.263 \mathrm{GPa}$, respectively. The lubricant is able to nucleate the hole for the squeeze-out at the lowest of these pressures, but at high pushing speed there is not enough time to exploit this possibility before the pressure is further increased.

It is interesting to observe what happens when the temperature is changed, particularly considering that in reality the microcontacts where the squeeze-out happens are likely to be much warmer than the environment, at least during sliding. The temperature is likely to speed up the mobility of the molecules, reducing the influence of the drag force of the walls. Moreover the larger thermal fluctuations should strongly favor the nucleation of the critical hole in the lubricant layer. Both effects tend to reduce the pressure needed to squeeze the lubricant. This is indeed the trend which we found and that is observed in Fig. 10. On the other hand, any difference $\Delta G$ between $n$-butane and isobutane in the free energy barriers for nucleation of squeeze-out should become less important as the temperature increases, as the ratio between the nucleation probabilities is mainly affected by the Boltzmann factor $\exp (-\beta \Delta G)$, which goes to 1 when $T \rightarrow \infty$.

Unfortunately the low temperature conditions are hard to analyze through simulations: the randomness of the single squeeze-out event is enhanced by the strong decrease of the Boltzmann factor. Actually the transition time for a nucleating process has an exponential distribution, where the standard deviation is equal to the average value. Thereafter the data of a single simulation become no more reliable and only an average over many independent simulations-not easily affordable due to computer time limitations-would show the real trend. We do not have enough statistics in Fig. 10 for a reliable comparison of the behaviors of $n$-butane and isobutane below room temperature.

Finally we consider the effect of lateral sliding of the walls with respect to each other. We ran some simulations (not shown) at different temperatures with squeezing speed and lateral speed both equal to $1 \mathrm{~m} / \mathrm{s}$, and we observed that the removal of the last monolayer happens almost simultaneously at the same pressure. The relative motion of the walls tends to take away the effect of the drag between walls and lubricant, which is indeed responsible of the higher lubricity of isobutane when squeezing fast. This is an important remark, since in many practical situations there is lateral sliding.

The significance of the temperature for the squeeze-out of the two different butanes is of utmost importance for future wear experiments. The results in Fig. 2 are all obtained at $298 \mathrm{~K}$. At this temperature and at the given sliding conditions it seems that isobutane is more easily squeezed out from the ball-disk contact than $n$-butane as the resulting wear is slightly lower for the latter one. In real injection pumps the temperature could be very low (at start-up) and very high (running conditions). The present simulation results imply that wear protecting properties of different molecules in the fuel may not react the same way to changes in temperature and sliding conditions. This means that a wear test may not reflect reality unless test conditions such as temperature and sliding velocity are varied. This new aspect of wear experiments should be looked into in the near future.

\section{SUMMARY AND CONCLUSIONS}

We have studied the properties of butane $\left(\mathrm{C}_{4} \mathrm{H}_{10}\right)$ lubricants confined between two approaching solids using a model that accounts for the curvature and elastic properties of the solid surfaces. We considered both linear $n$-butane and branched isobutane. In the case of the linear molecule well defined molecular layers develop in the lubricant film when the width is of the order of a few atomic diameters. The branched isobutane forms more disordered structures which permit it to stay liquidlike at smaller surface separations.

During squeezing the solvation forces show oscillations corresponding to the width of a molecule. At low speeds $(<0.1 \mathrm{~m} / \mathrm{s})$ the last layers of isobutane are squeezed out before those of $n$-butane. Since the (interfacial) squeezing velocity in most practical applications is very low when the lubricant layer has molecular thickness, one expect $n$-butane to be a better boundary lubricant than isobutane. This is consistent with wear experiments in which $n$-butane was shown to protect steel surfaces better than isobutane. On the other hand, with $n$-butane possessing lower viscosity at high pressures, our result refutes the view that squeeze-out should be harder for higher viscosities. At high squeezing velocity, the squeeze-out of the last monolayer of isobutane occurs at higher pressures than for $n$-butane. We interpret this as a kinetic effect resulting from the lateral corrugation barrier experienced by the molecules. As the temperature increases, 
the squeeze-out occurs at lower applied pressure for both $n$-butane and isobutane. This is the expected result based on the nucleation theory of squeeze-out. ${ }^{25,27}$

\section{ACKNOWLEDGMENTS}

Two of the authors (U.T. and I.M.S.) acknowledge support from IFF, FZ-Jülich and the hospitality and help of the staff during their research visits. One of the authors (I.M.S.) also acknowledges financial support from the European project AFFORHD. In Trieste, this work was also sponsored by MIUR COFIN No. 2003028141-007, MIUR COFIN No. 2004028238-002, MIUR FIRB RBAU017S8 R004, and MIUR FIRB RBAU01LX5H.

${ }^{1}$ B. N. J. Persson, Sliding Friction: Physical Principles and Applictions (Springer, Heidelberg, 2000).

${ }^{2}$ J. N. Israelachvili, Intermolecular and Surface Forces (Academic, London, 1995).

${ }^{3}$ Annual Book of ASTM Standards: D6079-97 5.03, 1355 (1998).

${ }^{4}$ ISO Standards ISO 12156-1 and 2 (1997).

${ }^{5}$ I. M. Sivebaek and S. C. Sorenson, Soc. Automot. Eng. [Spec. Publ.] SAE Tech. Paper 2000-01-2970 (2000).

${ }^{6}$ I. M. Sivebaek, S. C. Sorenson, and J. Jakobsen, Tribol. Lett. (to be published)

${ }^{7}$ I. M. Sivebaek, S. C. Sorenson, and J. Jakobsen, Soc. Automot. Eng. [Spec. Publ.] SAE Tech. Paper 2001-01-2013 (2001).
${ }^{8}$ I. M. Sivebaek, V. N. Samoilov, and B. N. J. Persson, J. Chem. Phys. 119, 2314 (2003)

${ }^{9}$ H. K. Christenson, J. Chem. Phys. 78, 6906 (1983).

${ }^{10}$ H. K. Christeenson, R. G. Horn, and J. N. Israelachvili, J. Colloid Interface Sci. 88, 79 (1982).

${ }^{11}$ J. Israelachvili, S. J. Kott, M. L. Gee, and T. A. Witten, Macromolecules 22, 4247 (1989).

${ }^{12}$ M. L. Gee, P. M. McGuiggan, and J. N. Israelachvili, J. Chem. Phys. 93, 1895 (1990)

${ }^{13}$ Y. Wang, K. Hill, and J. G. Harris, J. Chem. Phys. 100, 3276 (1994).

${ }^{14}$ M. Dijkstra, J. Chem. Phys. 107, 3277 (1997).

${ }^{15}$ H. Tamura, M. Yoshida, K. Kusakabe, C. Young-Mo, R. Miura, M. Kubo, K. Teraishi, A. Chatterjee, and A. Miyamoto, Langmuir 15, 7816 (1999).

${ }^{16}$ J. C. Wang and K. A. Fichthorn, J. Chem. Phys. 116, 410 (2002).

${ }^{17}$ S. T. Cui, P. T. Cummings, and H. D. Cochran, J. Chem. Phys. 114, 6464 (2001).

${ }^{18}$ A. M. Homola, J. N. Israelachvili, P. M. McGuiggan, and M. L. Gee, Wear 136, 65 (1990).

${ }^{19}$ D. P. Wei, H. A. Spikes, and S. Korcek, Tribol. Trans. 42, 813 (1999).

${ }^{20}$ W. I. Jorgensen, J. D. Madura, and C. J. Swenson, J. Am. Chem. Soc. 106, 6638 (1984)

${ }^{21}$ D. K. Dysthe, A. H. Fuchs, and B. Rousseau, J. Chem. Phys. 112, 7581 (2000).

${ }^{22}$ M. Mondello and G. S. Grest, J. Chem. Phys. 103, 7156 (1995).

${ }^{23}$ T. K. Xia, J. Ouyang, M. W. Ribarsky, and U. Landman, Phys. Rev. Lett. 69, 1967 (1992).

${ }^{24}$ S. M. Wetterer, D. J. Lavrich, T. Cummings, S. L. Bernasek, and G. Scoles, J. Phys. Chem. B 102, 9266 (1998).

${ }^{25}$ B. N. J. Persson and E. Tosatti, Phys. Rev. B 50, 5590 (1994).

${ }^{26}$ B. N. J. Persson and P. Ballone, J. Chem. Phys. 112, 9524 (2000).

${ }^{27}$ B. N. J. Persson and F. Mugele, J. Phys.: Condens. Matter 16, R295 (2004). 